\title{
Antígenos del sistema sanguíneo ABO como factor de riesgo para la gravedad de la infección por SARS-CoV-2
}

Carmen G. Torres-Alarcón, ${ }^{1 *}$ Antonio García-Ruíz, ${ }^{2}$ Cesar R. Cañete-Ibáñez, ${ }^{3}$ Irving I. Morales-Pogoda, ${ }^{4}$ Carlos M. Muñoz-Arce, ${ }^{5}$ Blanca E. Cid-Domínguez, ${ }^{1}$ Maribel Montalvo-Bárcenas, ${ }^{1}$ Guadalupe Maza-de la Torre, ${ }^{4}$ Chantal Sandoval-López, ${ }^{6}$ Emanuel Gaytán-Guzmán ${ }^{6}$ y José D. Correo- Zamora ${ }^{5}$

${ }^{1}$ Departamento de Banco de Sangre; ${ }^{2}$ Subdirección Técnica; ${ }^{3}$ Oficina COVID, Secretaría de la Defensa Nacional; ${ }^{4}$ Departamento de Medicina Crítica; ${ }^{5}$ Coordinación Hospitalaria de Donación y Trasplantes; ${ }^{6}$ Departamento de Enseñanza. Hospital Central Militar. Ciudad de México, México

\section{Resumen}

Introducción: Se desconoce si existe una influencia del sistema sanguíneo $A B O$ en susceptibilidad y gravedad de la enfermedad. Objetivo: Analizar si existe una asociación entre los antígenos del sistema ABO y la susceptibilidad y gravedad de la infección por SARS-CoV-2. Material y métodos: Se compararon las frecuencias de los antígenos del sistema ABO en 73 casos confirmados de infección por SARS-CoV-2 y 52 donadores clínicamente sanos. La gravedad de la infección se evaluó comparando la frecuencia de los antígenos por gravedad de la enfermedad y la mortalidad. Resultados: El riesgo de padecer infección por SARS-CoV-2 se incrementa en sujetos con antígeno A vs los no-A (OR=1.45; IC95 \%:1.061-1.921). EI fenotipo sanguíneo $O$ disminuye el riesgo de padecer infección por SARS-CoV-2 (OR=0.686; IC95 \%: 0.522-0.903). No se encontraron diferencias entre la gravedad de la enfermedad. En los pacientes graves, el riesgo de mortalidad se incrementó en sujetos con antígeno $A$ vs los no-A (OR=3.34; IC95 \%: 1.417-8.159). Conclusión: El grupo sanguíneo $A$ es un factor de riesgo para padecer infección por SARS-CoV-2, no así en la gravedad de la enfermedad, pero en los pacientes graves fue un factor de riesgo para la mortalidad.

PALABRAS CLAVE: Grupo sanguíneo ABO. Infección por SARS-CoV-2. COVID-19.

\section{Blood system ABO antigens as risk factor for severity of SARS-CoV-2 infection}

\begin{abstract}
Introduction: Whether there is an influence of the $A B O$ blood system on susceptibility to the disease and its severity is unknown. Objective: To analyze if there is an association between the $A B O$ blood system phenotypes and susceptibility to SARS-COV-2 infection and its severity. Material and methods: The frequency of $A B O$ antigens was compared in 73 confirmed cases of SARS-COV-2 infection and 52 clinically healthy donors. The severity of the infection was evaluated by comparing the frequency of antigens by severity of the disease and mortality. Results: The risk of SARS-CoV-2 infection is increased in subjects with antigen $A$ vs non- $A$ subjects (OR=1.45; $95 \%: 1.061-1.921)$. Blood phenotype $O$ decreases the risk of SARSCoV-2 infection (OR= 0.686; $95 \% \mathrm{Cl}$ : 0.522-0.903). No differences were found regarding disease severity. The mortality risk is increased in subjects antigen $A$ vs non-A (OR=3.34; 95\% IC:1.417-8.159). Conclusion: Blood group $A$ is a risk factor for SARS-CoV-2 infection, but not for disease severity, although in critically ill patients it is a risk factor for mortality.
\end{abstract}

KEY WORDS: ABO blood group. SARS-CoV-2 infection. COVID-19.

Correspondencia:

*Carmen G. Torres-Alarcón

E-mail: dragabytorresalarcon@ hotmail.com

0016-3813/๑ 2019 Academia Nacional de Medicina de México, A.C. Publicado por Permanyer. Este es un artículo open access bajo la licencia CC BY-NC-ND (http://creativecommons.org/licenses/by-nc-nd/4.0/).

Fecha de recepción: 17-07-2020

Fecha de aceptación: 17-08-2020

A.C. Publicado por Permanyer. Este es un artículo open access bajo la licencia 


\section{Introducción}

La infección causada por el coronavirus 2 del síndrome respiratorio agudo grave (SARS-CoV-2), Ilamada enfermedad por coronavirus 2019 (COVID-19), ha cobrado la vida de más de 549 mil personas en todo el mundo., México se ha ubicado como uno de los países con mayor número de muertes, superando a países como Italia y España. La COVID-19 tiene características clínicas variadas, que van desde casos asintomáticos, sintomatología leve, afectación moderada (neumonía) hasta casos graves, que se manifiestan con un síndrome de dificultad respiratoria aguda (SDRA), que conlleva una elevada mortalidad. La patogénesis de la COVID-19 grave es poco conocida, pero factores como la edad, el sexo y la presencia comorbilidades se han asociado a su mortalidad. ${ }^{3,4}$

En los últimos días ha crecido un interés en conocer si los antígenos del sistema sanguíneo ABO tienen un papel en la susceptibilidad de infección por SARSCoV-2 o en la gravedad de la enfermedad. Debido a que los antígenos del sistema $A B O$ se han asociado a otro tipo de enfermedades, ${ }^{5}$ como las oncológicas (cáncer de ovario, gástrico y cáncer de próstata) ${ }^{6} \mathrm{e}$ infecciosas, incluidas las ocasionadas por parásitos como Plasmodium falciparum y Plasmodium vivax, ${ }^{5,7}$ infecciones bacterianas como las causadas por Escherichia coli, Helicobacter pylor ${ }^{5,6}$ y también infecciones virales por parvovirus $\mathrm{B} 19^{8}$, virus de la hepatitis $B$, virus chikungunya y virus de Nilo, entre otras.

Los grupos sanguíneos son clasificados en sistemas, colecciones y antígenos de baja incidencia $(<1 \%) .{ }^{9} \mathrm{La}$ característica de un sistema es que se encuentra controlado en un solo locus genético; para el caso del sistema $A B O$, este se encuentra en el brazo largo del cromosoma 9 en la posición 34.2 (9q34.2). ${ }^{10}$ El grupo sanguíneo $A B O$ es el sistema más importante en medicina transfusional y en el trasplante de órganos. Los antígenos que lo forman (A, B) están presentes en otros tejidos, incluyendo endotelio, riñón, corazón, intestino y páncreas. ${ }^{11}$ Los antígenos del sistema ABO están formados por proteínas y carbohidratos unidos a proteínas o a lípidos, y consta de cuatro fenotipos determinados genéticamente: $A, B, O$ y $A B$, que muestran una diferencia en la expresión y cantidad de antígenos, en los eritrocitos y en secreciones. El grupo $\mathrm{ABO}$ también se caracteriza por la presencia o ausencia de anticuerpos naturales dirigidos hacia los antígenos A y B (Figura 1).

En vista de que se ha demostrado que el grupo sanguíneo $A B O$ tiene una influencia en enfermedades infecciosas, se desea conocer si también existe una asociación con el SARS-CoV-2.

\section{Material y métodos}

Se realizó un estudio de casos y controles, que incluyó a 73 pacientes que cumplieron con la definición operacional de caso confirmado de COVID-19. Todos los pacientes que se incluyeron contaban con una prueba de reacción en cadena de la polimerasa (PCR) de ARN viral de SARS-CoV-2 de hisopos nasofaríngeos positiva y recibieron atención en el Hospital Central Militar en el periodo del 23 de junio al 3 de julio del 20202. Para analizar el riesgo de padecer infección por SARS-CoV-2 y los fenotipos sanguíneos se comparó con un grupo control, formado por donadores clínicamente sanos de acuerdo a la NOM-253-SSA1-2012 ${ }^{12}$ que acudieron como donadores voluntarios al Banco de Sangre del Hospital Central Militar. Para el análisis entre la gravedad de presentación de la COVID-19 y los fenotipos sanguíneos, los pacientes se agruparon de acuerdo a la gravedad de la enfermedad con sintomatología leve y casos graves (clínicamente con SDRA). Se registró el fenotipo sanguíneo del sistema $A B O$ y el grupo $\mathrm{RH}$, los cuales fueron determinados por un sistema automatizado en el equipo Wadiana! (Grifold), por metodología de aglutinación en columna en tarjetas de gel (DG Gela). Se comparó las características demográficas como sexo, edad y las características clínicas de gravedad como presencia de intubación, ingreso a la Unidad de Terapia Intensiva y muerte. Los datos se expresaron en porcentajes, se realizaron comparaciones entre las frecuencias de los fenotipos sanguíneos y los grupos de estudio (donadores sanos versus pacientes COVID-19) y entre los pacientes con COVID-19 se realizaron comparaciones entre las frecuencias fenotípicas (ABO) y la gravedad de la enfermedad, presencia de intubación, ingreso a la unidad de terapia intensiva (UTI) y la presencia de muerte. Se realizaron tablas cruzadas utilizando la prueba de chi-cuadrada, se analizó el riesgo mediante la odds ratio (OR) y su intervalo de confianza del $95 \%$ (IC 95 $\%$ ), se consideró un valor de $p \leq 0.05$ como estadísticamente significativo. El análisis estadístico se realizó utilizando el Paquete Estadístico para Ciencias Sociales (SPSS), versión 25.0 (SPSS Inc., Chicago, IL, EE.UU.), con licencia para la Universidad Anáhuac Norte. La presente investigación se clasifica como investigación sin riesgo, de acuerdo con el Reglamento de la Ley General Investigación para la Salud de Salud en Materia de Investigación para la Salud. 


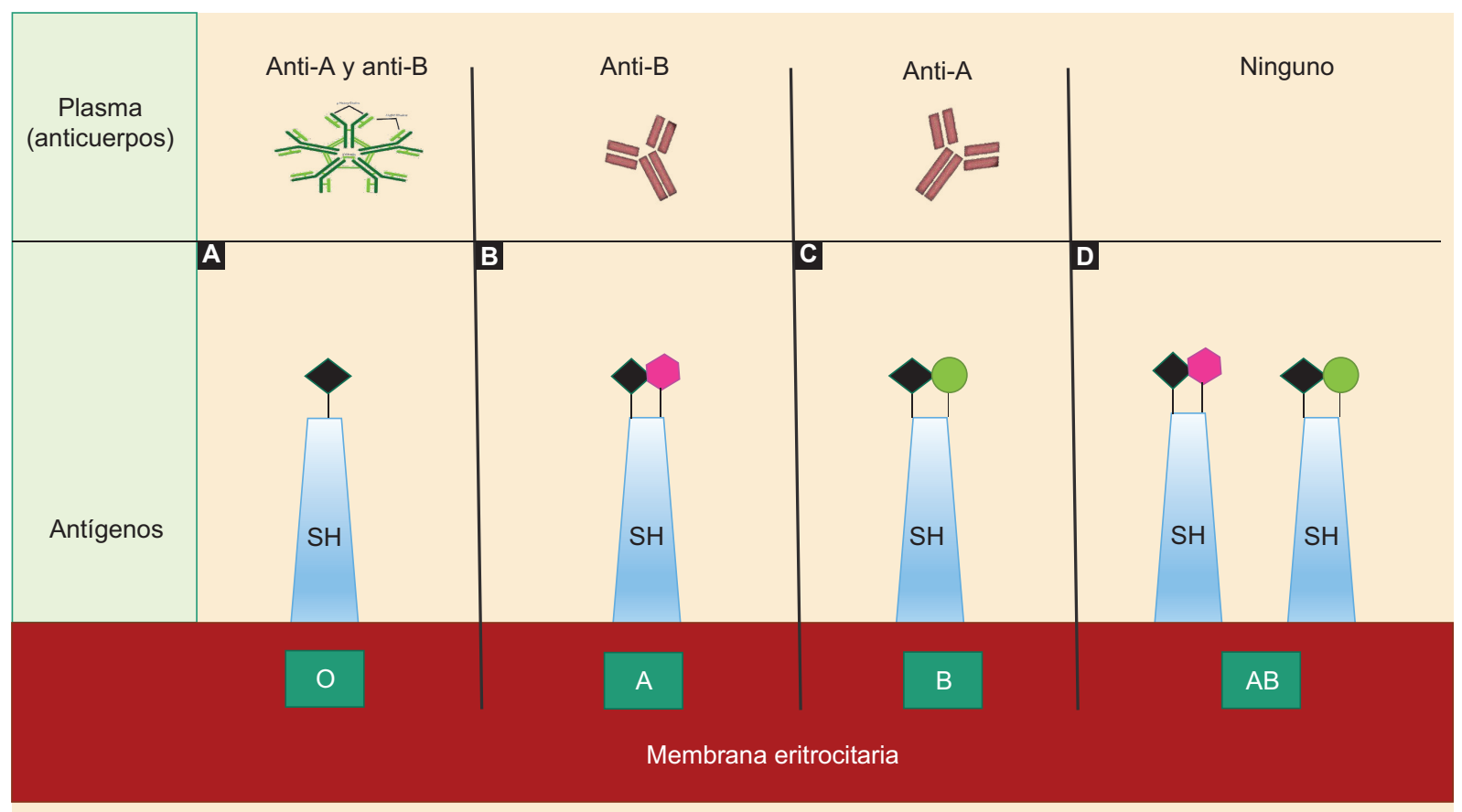

Figura 1. Esquema de los cuatro fenotipos sanguíneos del sistema $A B O$, las características de los antígenos en la membrana eritrocitaria y sus anticuerpos en el plasma. En la figura se observa una representación gráfica de los cuatro fenotipos del sistema ABO: en la parte basal en rojo se encuentra la membrana eritrocitaria y la manera en la que se expresan los dos antígenos del sistema ABO: el antígeno $A$ (hexágono rosa) y el antígeno $B$ (círculo verde). En la región superior de la imagen se muestra la presencia de los anticuerpos naturales del sistema $A B O$ en cada fenotipo sanguíneo. Todos los fenotipos expresan una sustancia precursora llamada "sustancia $H$ " que está representado por un trapecio azul y un rombo negro. A: fenotipo $O$, compuesto de la sustancia $H$ más fucosa (rombo negro). B: fenotipo $A$, compuesto de la sustancia $H$ más azúcar terminal acetilgalactosamina (hexágono rosa). C: fenotipo $B$, compuesto de la sustancia $H$ más azúcar terminal galactosa (círculo verde). D: fenotipo $A B$, compuesto de la sustancia $H$ más azucares terminales acetilgalactosamina (hexágono rosa) y galactosa (círculo verde).

\section{Resultados}

Las características demográficas de los pacientes con infección por SARS-CoV-2 y los controles se describen en la Tabla 1.

Al comparar la distribución por edad y sexo en el grupo de pacientes con COVID-19 y el grupo control no se encontró diferencia estadística en relación con el sexo, pero la edad mostró una diferencia, siendo mayor la edad de los pacientes con COVID-19 que el grupo control $(43.75 \pm 13.27$ versus $24.46 \pm 7.04$, respectivamente; $p<0.001$ ) (Figura 2).

La proporción del fenotipo sanguíneo $A$ en pacientes con infección por SARS-CoV-2 fue mayor que la de los controles sanos ( 24.7 versus $9.6 \% ; p=0.032$ ), mientras que la proporción del fenotipo sanguíneo $O$ en pacientes infectados con SARS-CoV-2 fue menor que la de los controles sanos (68.5 versus $86.5 \% ; p=0.02$ ).

Para conocer si existe una mayor susceptibilidad de infección por SARS-CoV-2 asociada a los antígenos del grupo sanguíneo $A B O$, se analizaron las diferencias en las frecuencias entre los portadores de los diferentes antígenos sanguíneos y se compararon con las de aquellos sin el antígeno, de tal manera que las comparaciones se realizaron en tres grupos: los individuos con antígenos $A$ versus no- $A, B$ versus no- $B$ y $\mathrm{O}$ versus No-O (Tabla 2).

Al comparar el grupo de pacientes con COVID-19 y su gravedad, solo se evidenció una diferencia en la edad, siendo mayor la edad de los pacientes con formas graves versus formas leves: de $51.38 \pm 11.67$ versus $34.52 \pm 8.35(p<0.001)$ (Figura 3$)$. No se identificaron diferencias en el sexo, fenotipo sanguíneo y grupo Rh (Tabla 3).

Se analizaron las frecuencias de los fenotipos sanguíneos y la necesidad de intubación, ingreso a una UTI y la presencia de muerte, identificando una diferencia en la frecuencia observadas en las personas que fallecieron y el fenotipo sanguíneo, siendo mayor en aquellos con fenotipo $A$ en comparación con los otros fenotipos sanguíneos (46.7 versus $15.2 \%$; $p=0.017)$. No se identificaron diferencias entre la necesidad de intubación o ingreso a la UTI y su fenotipo sanguíneo (Tabla 4).

Para conocer si existe un riesgo entre el desenlace (vivo/fallecido) y los antígenos del grupo sanguíneo 
Tabla 1. Características demográficas y grupo sanguíneo ABO en pacientes con enfermedad por coronavirus 2019 (COVID-19) versus controles sanos

\begin{tabular}{|c|c|c|c|c|c|}
\hline & Todos $n=125(100 \%)$ & COVID-19 n = $73(100 \%)$ & Control $n=52(100 \%)$ & $\chi^{2}$ & p \\
\hline $\begin{array}{l}\text { Edad } \\
\text { Media (DS) } \\
\text { (mín-máx) }\end{array}$ & $\begin{array}{c}35.7 \pm 14.6 \\
(18-71)\end{array}$ & $\begin{array}{c}43.75 \pm 13.27 \\
(23-71)\end{array}$ & $\begin{array}{c}24.46 \pm 7.04 \\
(18-62)\end{array}$ & $-7.978^{\star}$ & $<0.001$ \\
\hline $\begin{array}{l}\text { Sexo } \\
\text { Masculino } \\
\text { Femenino }\end{array}$ & $\begin{array}{l}103(82.4 \%) \\
22(17.6 \%)\end{array}$ & $\begin{array}{l}60(82.2 \%) \\
13(17.8 \%)\end{array}$ & $\begin{array}{c}43(82.7 \%) \\
9(17.3 \%)\end{array}$ & 0.005 & 0.942 \\
\hline $\begin{array}{l}\text { Grupo sanguíneo } \\
\text { A } \\
\text { B } \\
\text { O }\end{array}$ & $\begin{array}{c}23(18.4 \%) \\
7(5.6 \%) \\
95(76 \%)\end{array}$ & $\begin{array}{c}18(24.7 \%) \\
5(6.8 \%) \\
50(68.5 \%)\end{array}$ & $\begin{array}{c}5(9.6 \%) \\
2(3.8 \%) \\
45(86.5 \%)\end{array}$ & 5.525 & 0.063 \\
\hline $\begin{array}{l}\text { Grupos de estudio } \\
\text { A } \\
\text { no-A } \\
\text { B } \\
\text { no-B } \\
\text { O } \\
\text { no-O }\end{array}$ & $\begin{array}{c}23(18.4 \%) \\
102(81.6 \%) \\
7(5.6 \%) \\
118(94.4 \%) \\
95(76 \%) \\
30(24 \%)\end{array}$ & $\begin{array}{c}18(24.7 \%) \\
55(75.3 \%) \\
5(6.8 \%) \\
68(93.2 \%) \\
50(68.5 \%) \\
23(31.5 \%)\end{array}$ & $\begin{array}{c}5(9.6 \%) \\
47(90.4 \%) \\
2(3.8 \%) \\
50(96.2 \%) \\
45(86.5 \%) \\
7(13.5 \%)\end{array}$ & $\begin{array}{l}4.576 \\
0.518 \\
5.422\end{array}$ & $\begin{array}{l}0.032 \\
0.472 \\
0.02\end{array}$ \\
\hline $\begin{array}{l}\text { RH } \\
\text { Positivo } \\
\text { Negativo }\end{array}$ & $\begin{array}{c}123(98.4 \%) \\
2(1.6)\end{array}$ & $\begin{array}{c}71(97.3 \%) \\
2(2.7 \%)\end{array}$ & $\begin{array}{c}52(100 \%) \\
0(0 \%)\end{array}$ & 2.19 & 0.139 \\
\hline
\end{tabular}

DS = desviación estándar.

* Prueba U de Mann-Whitney.

Tabla 2. Análisis de riesgo entre los fenotipos sanguíneos en pacientes con enfermedad por coronavirus 2019 (COVID-19) versus controles sanos

\begin{tabular}{l|c|c|c|c|}
$\begin{array}{l}\text { Grupos de } \\
\text { comparación }\end{array}$ & COVID-19 OR & $($ IC 95\%) & $\chi^{2}$ & $p$ \\
\hline A versus no-A & 1.451 & $(1.061-1.921)$ & 4.576 & 0.032 \\
\hline B versus no-B & 1.239 & $(0.757-2.03)$ & 0.518 & 0.472 \\
\hline $\begin{array}{l}\text { O versus no-O } \\
\text { OR = odds ratio; IC = intervalo de confianza. }\end{array}$ & 0.686 & $(0.522-0.903)$ & 5.422 & 0.020 \\
\hline
\end{tabular}

$\mathrm{ABO}$, se analizaron las diferencias entre los diferentes antígenos sanguíneos y aquellos sin el antígeno (A versus no-A, B versus no-B y $O$ versus No-O), se observó lo que se muestra en la Tabla 5.

\section{Discusión}

La diferencia en la expresión de antígenos del grupo sanguíneo $A B O$ puede incrementar la susceptibilidad a muchas infecciones. Algunos grupos sanguíneos están relacionados con receptores de diferentes microrganismos, incluidos los virus, y además algunos grupos sanguíneos pueden modificar la inmunidad innata como respuesta a la infección.

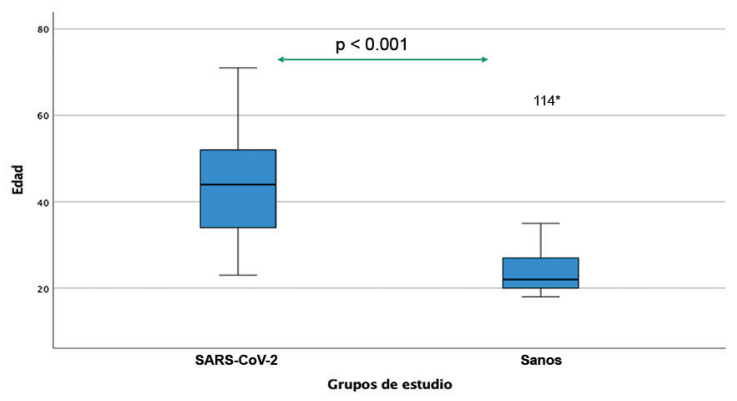

Figura 2. Distribución de la edad en los grupos de estudio (pacientes con infección por coronavirus 2 del síndrome respiratorio agudo grave [SARS-CoV-2] versus individuos sanos). En esta gráfica de cajas y bigotes se muestra la diferencia entre la distribución de la edad en los dos grupos de estudio: los límites de edad en los pacientes con infección por SARS-CoV-2 fue de 23 y 71 años y en los controles de 18 y 62 años (bigotes de cada una de las cajas), la mediana fue de 54 años en los pacientes con infección por SARS-CoV-2 y de 23 años en los controles (línea que divide a la caja). Se aplicó la prueba U de Mann-Whitney. *El 114 es un valor atípico que representa la edad de un paciente del grupo control.

Los antígenos del sistema $\mathrm{ABO}$ son un conjunto de glucolípidos y glucopéptidos celulares secretados y distribuidos por todo el cuerpo. El sistema sanguíneo ABO se ha asociado a la susceptibilidad por otros coronavirus. En la epidemia causa por el SARS-CoV-1 
Tabla 3. Características demográficas y grupo sanguíneo ABO en pacientes con enfermedad por coronavirus 2019 (COVID-19) versus grave

\begin{tabular}{|c|c|c|c|c|c|}
\hline & Todos $n=73(100 \%)$ & COVID-19 leve $n=33(100 \%)$ & COVID-19 grave $n=40(100 \%)$ & $\chi^{2}$ & p \\
\hline $\begin{array}{l}\text { Edad } \\
\text { Media (DS) } \\
\text { (mín-máx) }\end{array}$ & $\begin{array}{c}43.75 \pm 13.27 \\
(23-71)\end{array}$ & $\begin{array}{c}34.52 \pm 8.35 \\
(23-48)\end{array}$ & $\begin{array}{c}51.38 \pm 11.67 \\
(26-71)\end{array}$ & $5.398^{*}$ & $<0.001$ \\
\hline $\begin{array}{l}\text { Sexo } \\
\text { Masculino } \\
\text { Femenino }\end{array}$ & $\begin{array}{l}60(82.2 \%) \\
13(17.8 \%)\end{array}$ & $\begin{array}{c}29(87.9 \%) \\
4(12.1 \%)\end{array}$ & $\begin{array}{c}31(77.5 \%) \\
9(22.5 \%)\end{array}$ & 1.331 & 0.249 \\
\hline $\begin{array}{l}\text { Fenotipo sanguíneo } \\
\text { A } \\
\text { B } \\
\text { O }\end{array}$ & $\begin{array}{c}18(24.7 \%) \\
5(6.8 \%) \\
50(68.5 \%)\end{array}$ & $\begin{array}{c}9(27.3 \%) \\
2(6.1 \%) \\
24(72.7 \%)\end{array}$ & $\begin{array}{l}11(27.5 \%) \\
3(7.5 \%) \\
26(65 \%)\end{array}$ & 0.502 & 0.778 \\
\hline $\begin{array}{l}\text { Rh } \\
\text { Positivo } \\
\text { Negativo }\end{array}$ & $\begin{array}{l}71(97.3 \%) \\
2(2.7 \%)\end{array}$ & $\begin{array}{c}32(97 \%) \\
1(3 \%)\end{array}$ & $\begin{array}{c}39(97.5 \%) \\
1(2.5 \%)\end{array}$ & 1.019 & 0.89 \\
\hline
\end{tabular}

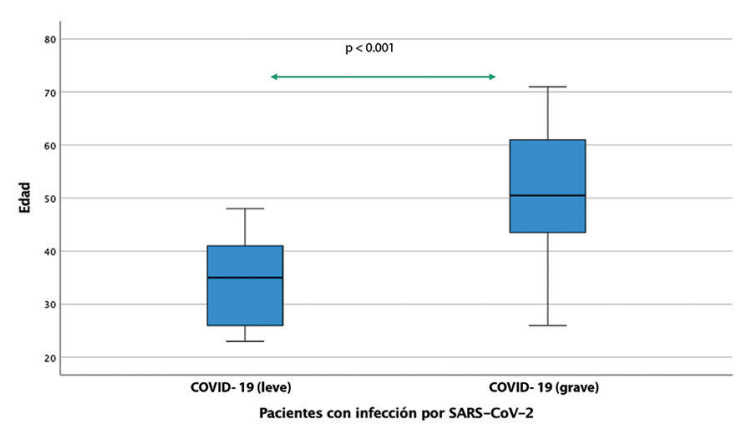

Figura 3. Distribución de la edad en los grupos de estudio (pacientes con COVID-19 leve versus grave). En esta gráfica de cajas y bigotes se muestra las diferencia en la distribución de la edad en los dos grupos de estudio: los límites de edad en los pacientes con infección por SARS-CoV-2 leve fue de 23 y 48 años y en aquellos con presentación grave de 26 y 71 años (bigotes de cada una de las cajas); la mediana en cada uno de los grupos (línea que divide a la caja) fue de 36 años en el grupo de la presentación leve y de 51 años en las formas graves. Se aplicó la prueba U de Mann-Whitney. COVID-19 = enfermedad por coronavirus 2019; SARS-CoV-2 = coronavirus 2 del síndrome respiratorio agudo grave.

(2002), en Hong Kong se demostró una asociación entre la transmisión de la enfermedad y el sistema $A B O$, además de que los individuos del grupo $O$ fueron más resistentes a la infección $(\mathrm{OR}=0.18$; IC 95 $\%$ : 0.04-0.81; $p=0.003) .{ }^{13}$

Ellinghaus et al. realizaron un análisis de asociación del genoma completo para identificar posibles factores genéticos involucrados en el desarrollo de la COVID19 y demostraron que existe una asociación entre un grupo de genes que se encuentran en los cromosomas 3 y 9 (locus 3p21.3 y 9q34.2) que coincide con el locus del sistema sanguíneo ABO. ${ }^{14}$ En su investigación concluyen que los pacientes con fenotipo $A$ tienen mayor riesgo de infección por SARS-CoV-2 comparado con otros grupos sanguíneos $(\mathrm{OR}=1.45$; IC $95 \%: 1.20-1.75 ; p=1.48 \times 10^{-4}$ ) y un efecto protector para el fenotipo sanguíneo $\mathrm{O}$, en comparación con los otros grupos sanguíneos (OR $=0.65$; IC $95 \%$ : 0.53$\left.0.79 ; p=1.06 \times 10^{-5}\right)$. En sus resultados genéticos se confirma que el grupo sanguíneo A se asocia con riesgo de adquirir COVID-19.

Esta investigación demuestra que el riesgo de padecer infección por SARS-CoV-2 se incrementa en $45.1 \%$ veces más $(\mathrm{OR}=1.451$; IC $95 \%$ : 1.061-1.921; $p=0.032$ ) en sujetos del grupo sanguíneo A comparado con los que tienen otro grupo sanguíneo. Estos resultados son similares a los descrito por Wu et al., ${ }^{15}$ quienes reportaron un incremento del $54 \%$ (OR $=1.54$; IC $95 \%$ : 1.222-2.104; $p=0.006$ ) en el riesgo de padecer infección por SARS-CoV-2 en sujetos de grupo A versus no-A. Otros estudios, como el realizado por Göker et al. ${ }^{16}$ también reportaron el riesgo para este grupo sanguíneo, con un aumento de dos veces más OR (OR = 2.1; IC $95 \%$ : 1.5-2.9; $p<0.001)$.

Al comparar a los pacientes por gravedad y por la necesidad de intubación, los pacientes con COVID-19 (leve versus grave) y su fenotipo sanguíneo, no se encontraron diferencias entre la gravedad de la enfermedad, ni a la intubación y/o al ingreso a la UTI. Nuestros resultados son similares a lo descrito por Göker et al. ${ }^{16}$ y Zietz et al. ${ }^{17}$ quienes no encontraron asociación entre el grupo sanguíneo y la intubación y/o el ingreso a la UTI. 
Tabla 4. Frecuencias entre los fenotipos sanguíneos y características de gravedad en la enfermedad (desenlace, intubados, ingreso a unidad de terapia intensiva de adultos [UTIA])

\begin{tabular}{|c|c|c|c|c|c|c|}
\hline & \multicolumn{6}{|c|}{ Fenotipo sanguíneo } \\
\hline & Total & $A n=18$ & $B n=5$ & $0 n=44$ & $\chi^{2}$ & $p$ \\
\hline $\begin{array}{l}\text { Desenlace }(n=66) \\
\text { Vivo } \\
\text { Fallecido }\end{array}$ & $\begin{array}{l}52(78.8 \%) \\
14(21.2 \%)\end{array}$ & $\begin{array}{l}8(53.3 \%) \\
7(46.7 \%)\end{array}$ & $\begin{array}{c}5(100 \%) \\
0(0 \%)\end{array}$ & $\begin{array}{c}39(84.8 \%) \\
7(15.2 \%)\end{array}$ & 8.151 & 0.017 \\
\hline $\begin{array}{l}\text { Intubados }(n=73) \\
\text { No } \\
\text { Sí }\end{array}$ & $\begin{array}{l}31(42.5 \%) \\
42(57.5 \%)\end{array}$ & $\begin{array}{c}10(55.6 \%) \\
8(44.4 \%)\end{array}$ & $\begin{array}{l}3(60 \%) \\
2(40 \%)\end{array}$ & $\begin{array}{l}18(36 \%) \\
32(64 \%)\end{array}$ & 2.747 & 0.253 \\
\hline $\begin{array}{l}\text { Ingreso UTIA }(n=73) \\
\text { Sí } \\
\text { No }\end{array}$ & $\begin{array}{c}9(45 \%) \\
11(55 \%)\end{array}$ & $\begin{array}{l}9(50 \%) \\
9(50 \%)\end{array}$ & $\begin{array}{l}3(60 \%) \\
2(40 \%)\end{array}$ & $\begin{array}{l}16(32 \%) \\
34(68 \%)\end{array}$ & 2.877 & 0.237 \\
\hline
\end{tabular}

Tabla 5. Análisis de riesgo de mortalidad y los fenotipos sanguíneos

\begin{tabular}{|c|c|c|c|c|}
\hline $\begin{array}{l}\text { Grupos de } \\
\text { comparación }\end{array}$ & COVID-19 OR & (IC 95\%) & $\chi^{2}$ & $p$ \\
\hline A versus no-A & 3.34 & $(1.417-8.159)$ & 7.526 & 0.006 \\
\hline$B$ versus no-B & ------- & ---------- & --------- & ------- \\
\hline O versus no-O & 0.435 & $(0.176-1.077)$ & 3.264 & 0.071 \\
\hline
\end{tabular}

Con respecto a la mortalidad, se observó que en los pacientes con COVID-19 el riesgo de morir por la enfermedad se incrementa tres veces más $(\mathrm{OR}=3.4$; IC $95 \%$ : 1.417-8.159; $p=0.006$ ) en sujetos de grupo sanguíneo A. Nuestros resultados son diferentes a los descritos por Zietz y Tatonetti, ${ }^{17}$ quienes en una muestra de 1,559 pacientes de tres regiones geográficas (New York, Wuhan y Shenzhen) no identificaron diferencias estadísticas entre los pacientes fallecidos y los vivos. Es importante mencionar que en su investigación el fenotipo sanguíneo fue obtenido de los expedientes electrónicos de los pacientes, por lo tanto, la información por ese medio puede estar sujeta a un sesgo de información.

Esta investigación confirma que existe una participación del sistema $A B O$ en la susceptibilidad de padecer infección por SARS-CoV-2 y en la mortalidad por la COVID-19, aunque aún no es claro el mecanismo al cual pueda deberse. Existen dos posibles mecanismos biológicos que puedan explicar esta asociación; el primero puede estar relacionado con los anticuerpos naturales del sistema $A B O$, en este caso, el efecto protector en las personas con fenotipo sanguíneo $O$ se explica por la presencia de anticuerpos anti-A. Por el contrario, las personas con fenotipo A, que carecen de este anticuerpo, serían los más vulnerables a infectarse. Este mecanismo se ha descrito dada la similitud entre los sacáridos de la membrana de algunos microorganismos y los sacáridos de los antígenos " $A$ " y "B", por lo que no sorprendería que este fenómeno ocurra también en la infección por el SARS-CoV-2. En investigaciones con otros coronavirus como el SARSCoV-1, la adhesión del virus por la proteína $S$ a las células diana con receptor de la enzima convertidora de angiotensina 2 fue inhibida específicamente por un anticuerpo monoclonal anti-A, lo que indica que estos anticuerpos pueden bloquear la interacción entre el virus y el receptor, proporcionando una protección. ${ }^{18}$ Si esta hipótesis es cierta, esto puede explicar la mayor susceptibilidad de los pacientes en edades mayores (Figuras 2 y 3), ya que los títulos de anticuerpos anti-A y anti-B sufren una caída de su titulación relacionada con la edad. Desconocemos el papel específico que pueda tener la presencia del antígeno A y sus variantes en la infección por SARS-CoV-2, incluso si esta asociación pueda deberse a la relación de los antígenos A y B con otros sistemas sanguíneos, por lo que otros factores deben investigarse.

\section{Conclusión}

El fenotipo sanguíneo A proporciona un riesgo de padecer la COVID-19. En esta investigación, que incluyó a un grupo de pacientes con sintomatología leve, al compararlos con los pacientes con COVID-19 grave no se demostró asociación entre los antígenos del sistema $A B O$ y la gravedad de la enfermedad, ni 
con la probabilidad de la intubación o el ingreso a la UTI, pero sí en la mortalidad por la COVID-19.

Se sugiere que las personas que se conozcan con grupo sanguíneo A fortalezcan la protección para reducir el riesgo de infección por el SARS-CoV-2 y en los hospitales se identifique a los pacientes con este grupo sanguíneo con la finalidad de que tengan una vigilancia médica más estrecha.

\section{Limitaciones}

Una de las limitaciones de esta investigación es el tamaño de la muestra, sin embargo, una de sus fortalezas es contar con los fenotipos sanguíneos de cada uno de los pacientes y donadores que se incluyeron en el estudio.

\section{Agradecimientos}

A todos los profesionales de la salud, médicos, enfermeras de las salas COVID, químicos y técnicos del Banco de Sangre del Hospital Central Militar.

\section{Conflicto de intereses}

Los autores declaran no tener conflicto de intereses alguno.

\section{Financiamiento}

Los autores no recibieron patrocinio para llevar a cabo este artículo.

\section{Responsabilidades éticas}

Protección de personas y animales. Los autores declaran que para esta investigación no se han realizado experimentos en seres humanos ni en animales.

Confidencialidad de los datos. Los autores declaran que han seguido los protocolos de su centro de trabajo sobre la publicación de datos de pacientes.
Derecho a la privacidad y consentimiento informado. Los autores declaran que en este artículo no aparecen datos de pacientes.

\section{Bibliografía}

1. Alocución de apertura del Director General de la OMS en la rueda de prensa sobre la COVID-19 celebrada el 11 de marzo de 2020 [Internet]. Organización Mundial de la Salud, Discursos del Director General de la OMS; 2020. Disponible en: https://www.who.int/es/director-general/speeches/detail/who-directorgeneral-s-opening-remarks-at-the-media-briefing-on-covid-19---11-march-2020

2. Covid-19 Dashboard by Center for System Science and Engineering (CSSE) at Johns Hopkins University [Internet]. Johns Hopkins University of Medicine, Coronavirus Resourse Center; 2020. Disponible en: https://coronavirus.jhu.edu/map.html

3. Manejo clínico de la infección respiratoria aguda grave presuntamente causada por el nuevo coronavirus (2019-nCoV). Orientaciones provisionales 28 de enero de 2020 [Internet]. Organización Mundial de la Salud; 28 de enero de 2020. Disponible en: https://apps.who.int/iris/handle/10665/330938

4. Clinical management of severe acute respiratory infection when $\mathrm{CO}$ VID-19 is suspected (v1.2) [Internet]. World Health Organization; 2020. Disponible en: https://www.who.int/publications-detail/clinical-management-of-severe-acute-respiratory-infection-when-novel-coronavirus-(ncov)-infection-is-suspected

5. Cooling L. Blood groups in infection and host susceptibility. Clin Microbiol Rev. 2015;28(3):801-70.

6. González-Ordóñez ÁJ. Grupos sanguíneos y enfermedad. Med Clin (Barc). 2005;125(10):382-8.

7. Toure O, Konate S, Sissoko S, Niangaly A, Barry A, Sall AH, et al, Candidate polymorphisms and severe malaria in a Malian population. PLoS One. 2012;7(9):5-10

8. Rosell R, Gómez-Codina J, Camps C, Maestre JA, Padille J, Cantó A, et al. A randomized trial comparing preoperative chemotherapy plus surgery with surgery alone in patients with non-small-cell lung cancer. $\mathrm{N}$ Engl J Med. 1994;330(3):153-8.

9. Armstrong B, Smart E. Blood group systems. ISBT Science Series [Internet]. 2008;3:93-109. Disponible en: https://onlinelibrary.wiley.com/doi/ epdf/10.1111/j.1751-2824.2008.00189.x

10. Yamamoto $F$. Evolutionary divergence of the $A B O$ and GBGT1 genes specifying the $\mathrm{ABO}$ and FORS blood group systems through chromosomal rearrangements. Sci Rep. 2017;7(1):1-10.

11. Yamamoto F, Cid E, Yamamoto M, Saitou N, Bertranpetit J, Blancher A. An integrative evolution theory of histo-blood group $A B O$ and related genes. Sci Rep. 2014:4:1-12.

12. Gobierno de México, Secretaría de salud. NOM-253-SSA1-2012, Para la disposición de sangre humana y sus componentes con fines terapéuticos. Diario Oficial; 2014. pp. 1-7.

13. Cheng Y, Cheng G, Chui CH, Lau FY, Chan PK, Ng MH, et al. ABO blood group and susceptibility to severe acute respiratory syndrome. JAMA. 2005;293(12):1450-1.

14. Severe Covid-19 GWAS Group, Ellinghaus D, Degenhardt F, Bujanda L, Buti M, Albillos A, Invernizzi P, et al. Genomewide association study of severe Covid-19 with respiratory failure. N Engl J Med. 2020;383(16):1522-34.

15. Wu Y, Feng Z, Li P, Yu Q. Relationship between $A B O$ blood group distribution and clinical characteristics in patients with COVID-19. Clin Chim Acta. 2020;509:220-3.

16. Göker $H$, Aladağ-Karakulak $E$, Demiroğlu $H$, Ayaz $C M$, Büyükașik $Y$, Inkaya AC, et al. The effects of blood group types on the risk of COVID-19 infection and its clinical outcome. Turkish J Med Sci. 2020;50(4):679-83.

17. Zietz M, Tatonetti NP. Testing the association between blood type and COVID-19 infection, intubation, and death. medRxiv. $2020 \mathrm{Apr}$ 11:2020.04.08.20058073. doi: 10.1101/2020.04.08.20058073. Preprint.

18. Guillon P, Clément M, Sébille V, Rivain JG, Chou CF, Ruvoën-Clouet N, et al. Inhibition of the interaction between the SARS-CoV Spike protein and its cellular receptor by anti-histo-blood group antibodies. Glycobiology. 2008;18(12):1085-93. 